\title{
Recent charm results from Belle
}

\section{Longke $\mathrm{Li}^{*}$}

On behalf of the Belle Collaboration

University of Cincinnati,

Cincinnati, Ohio 45221, U.S.

E-mail: lilk@ucmail.uc.edu

Recent charm results from Belle experiment are presented in this proceedings, including (1) measurement of mixing parameter $y_{C P}=\left(0.96 \pm 0.91 \pm 0.62_{-0.00}^{+0.17}\right) \%$ in $C P$-odd decay for the first time, (2) the first Dalitz-plot analysis of $D^{0} \rightarrow K^{-} \pi^{+} \eta$, (3) measurement of branching fractions of $\Lambda_{c}^{+} \rightarrow \eta \Lambda^{0} \pi^{+}$and $\eta \Sigma^{0} \pi^{+}$and intermediate processes $\Lambda_{c}^{+} \rightarrow\left[\Lambda(1670) \rightarrow \eta \Lambda^{0}\right] \pi^{+}$ and $\Lambda_{c}^{+} \rightarrow \eta \Sigma(1385)^{+}$relative to $\Lambda_{c}^{+} \rightarrow p K^{-} \pi^{+}: 0.293 \pm 0.003 \pm 0.014,0.120 \pm 0.006 \pm 0.006$, $(5.54 \pm 0.29 \pm 0.73) \times 10^{-2}$, and $0.192 \pm 0.006 \pm 0.016$, respectively, and (4) first determination of the spin and parity of a charmed-strange baryon $\Xi_{c}(2970)^{+}$which is consistent with the HQSS prediction for $J^{P}\left(s_{l}\right)=1 / 2^{+}(0)$.

40th International Conference on High Energy physics - ICHEP2020

July 28 - August 6, 2020

Prague, Czech Republic (virtual meeting)

${ }^{*}$ Speaker 


\section{Introduction to Belle at KEKB}

KEKB [1] is an asymmetric-energy $e^{+} e^{-}$collider operating at and near $\Upsilon(4 S)$ mass peak. As the only detector installed in KEKB, Belle detector has a good performance on momentum and vertex resolution, $K / \pi$ separation etc. A detailed description of the Belle detector can be found elsewhere [2]. It has been ten years since the final full data set $\left(\sim 1 \mathrm{ab}^{-1}\right)$ was accumulated, however, fruitful results on physics are lasting to be produced. Here we select some recent charm results from Belle to present in this proceedings.

\section{Charm-mixing parameter $y_{C P}$ in $D^{0} \rightarrow K_{S}^{0} \omega$}

The mixing parameter $y_{C P}$ is measured in $D^{0}$ decays to the $C P$-odd final state $K_{S}^{0} \omega$ for the first time [3]. Considering mixing parameters $|x|$ and $|y| \ll 1$, the decay-time dependence of $D^{0}$ to a $C P$ eigenstate is approximately exponential, $d \Gamma / d t \propto e^{-\Gamma\left(1+\eta_{f} y_{c p}\right) t}$ where $\eta_{f}=+1(-1)$ for $C P$-even (-odd) decays. Along with the decay rate in flavored eigenstate decays $d \Gamma / d t \propto e^{-\Gamma t}$, the $y_{C P}$ is determined by the decay proper-time value with the formula $y_{C P}=1-\frac{\tau\left(D^{0} \rightarrow K^{-} \pi^{+}\right)}{\tau\left(D^{0} \rightarrow K_{S}^{0} \omega\right)}$, where $D^{0} \rightarrow K^{-} \pi^{+}$is the chosen normalization mode with flavor eigenstate final state.

Based on the full Belle data sample of $976 \mathrm{fb}^{-1}$, we obtain 91 thousands of $D^{0} \rightarrow K_{S}^{0} \omega$ and 1.4 millions of reference mode $D^{0} \rightarrow K^{-} \pi^{+}$in $M-\Delta M$ signal region, where $M$ is the invariant mass of reconstructed $D^{0}$ and $\Delta M$ is the mass difference of reconstructed $D^{*+}$ and $D^{0}$. Using unbinned maximum-likelihood fits for lifetime on these two samples with high purities, the proper decay-time of $D^{0}$ is determined as $\tau_{K_{S}^{0} \omega}=(410.47 \pm 3.73)$ fs and $\tau_{K \pi}=(406.53 \pm 0.57)$ fs, as shown in Fig. 1 . Thus, we calculate $y_{C P}=\left(0.96 \pm 0.91 \pm 0.62_{-0.00}^{+0.17}\right) \%$, where the first uncertainty is statistical, the second is systematic due to event selection and background, and the last is due to possible presence of CP-even decays in the data sample. This $y_{C P}$ result is consistent with the world average value. In the future, comparing more precise measurements of $y_{C P}$ with that of $y$ may test the SM precisely or reveal new physics effects in the charm system.

\section{Dalitz-plot analysis of $D^{0} \rightarrow K^{-} \pi^{+} \eta$ decays}

The understanding of hadronic charmed-meson decay is theoretically challenging due to the significant non-perturbative contributions, and input from experimental measurements thus plays an important role. A Dalitz-plot analysis of $D^{0} \rightarrow K^{-} \pi^{+} \eta$ is performed for the first time at Belle based on $953 \mathrm{fb}^{-1}$ of data [4]. Using a $M-Q$ two-dimensional fit where $M$ is the invariantmass of reconstructed $D^{0}$ meson, $M=M\left(K^{+} \pi^{-} \eta\right)$, and $Q$ is the released energy of $D^{*+}$ decay, $Q=M\left(K^{-} \pi^{+} \eta \pi_{s}\right)-M-m_{\pi_{s}}$, a signal yield of $105197 \pm 990$ is obtained in the signal region of $1.85 \mathrm{GeV} / c^{2}<M<1.88 \mathrm{GeV} / c^{2}$ and $5.35 \mathrm{MeV} / c^{2}<Q<6.35 \mathrm{MeV} / c^{2}$ with a high purity $(94.6 \pm 0.9) \%$. The Dalitz plot is well described by a combination of the six resonant decay channels $\bar{K}^{*}(892)^{0} \eta, K^{-} a_{0}(980)^{+}, K^{-} a_{2}(1320)^{+}, \bar{K}^{*}(1410)^{0} \eta, K^{*}(1680)^{-} \pi^{+}$and $K_{2}^{*}(1980)^{-} \pi^{+}$, together with $K \pi$ and $K \eta$ S-wave components, as shown in Fig. 2. The dominant contributions to the decay amplitude arise from $\bar{K}^{*}(892)^{0}, a_{0}(980)^{+}$and the $K \pi$ S-wave component. The $K \eta$ $\mathrm{S}$-wave component, including $K_{0}^{*}(1430)^{-}$, is observed with a statistical significance of more than 

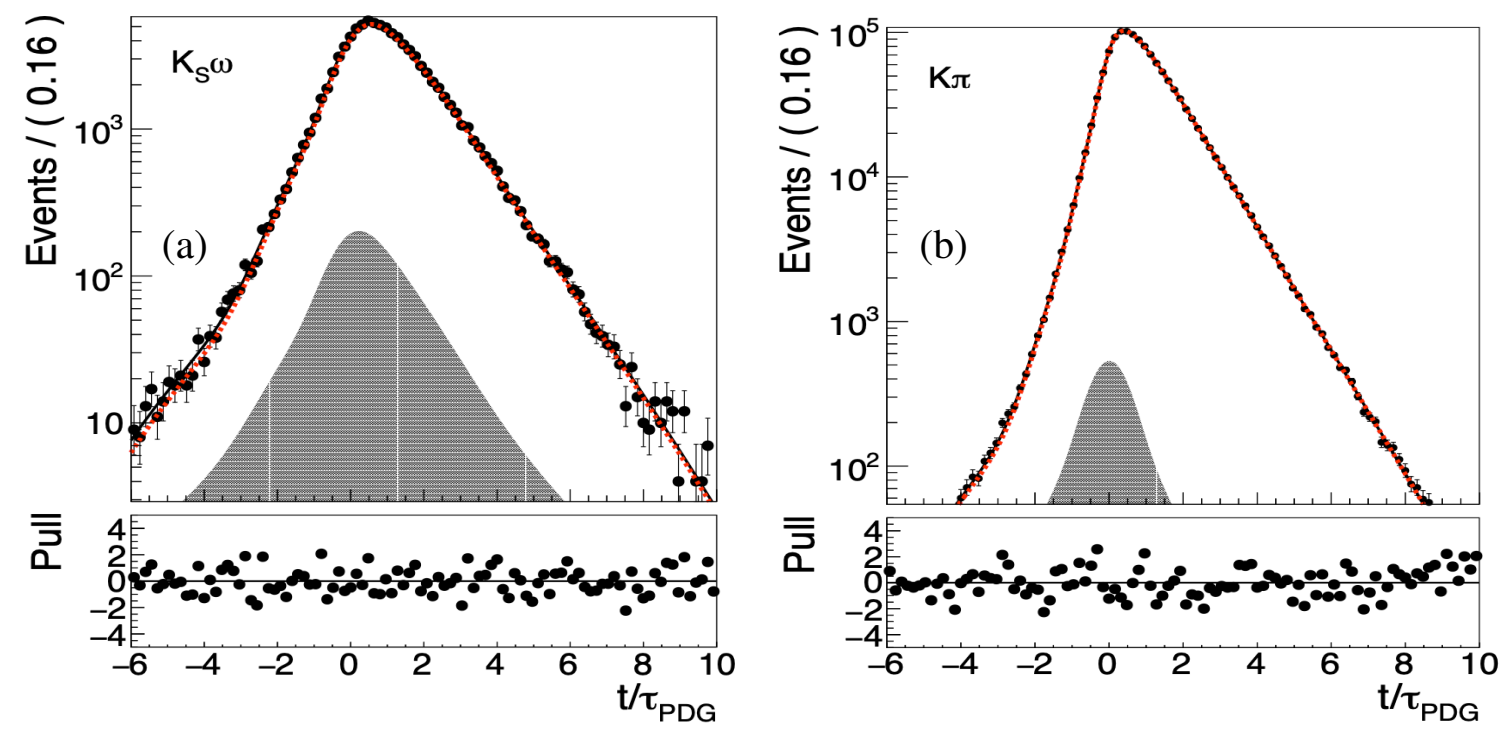

Figure 1: The fit of $D^{0}$ proper lifetime: (a) $D^{0} \rightarrow K_{S}^{0} \omega$ and (b) $D^{0} \rightarrow K^{-} \pi^{+}$. The dashed red curves are the signal contribution, and the shaded surfaces beneath are the background estimated from $M-\Delta M$ sidebands.

$30 \sigma$, and the decays $K^{*}(1680)^{-} \rightarrow K^{-} \eta$ and $K_{2}^{*}(1980)^{-} \rightarrow K^{-} \eta$ are observed for the first time and have statistical significances of $16 \sigma$ and $17 \sigma$, respectively.

We extract the signal yield from the $D^{0}$ invariant mass distribution in $1.78 \mathrm{GeV} / c^{2}<M<$ $1.94 \mathrm{GeV} / c^{2}$ and $|Q-5.85|<1.0 \mathrm{MeV} / c^{2}$, and obtain for the first time the branching ratio $\frac{\mathcal{B}\left(D^{0} \rightarrow K^{-} \pi^{+} \eta\right)}{\mathcal{B}\left(D^{0} \rightarrow K^{-} \pi^{+}\right)}=0.500 \pm 0.002$ (stat) \pm 0.020 (syst) $\pm 0.003\left(\mathcal{B}_{\text {PDG }}\right.$, which corresponds to $\mathcal{B}\left(D^{0} \rightarrow\right.$ $\left.K^{-} \pi^{+} \eta\right)=(1.973 \pm 0.009$ (stat) \pm 0.079 (syst $\left.) \pm 0.018\left(\mathcal{B}_{\mathrm{PDG}}\right)\right) \%$. Then utilizing the world average branching fractions of intermediate resonant decays, the relative branching ratio $\frac{\mathcal{B}\left(K^{*}(1680)^{-} \rightarrow K^{-} \eta\right)}{\mathcal{B}\left(K^{*}(1680)^{-} \rightarrow K^{-} \pi^{0}\right)}$ is determined to be $0.11 \pm 0.02$ (stat $)_{-0.04}^{+0.06}$ (syst) $\pm 0.04\left(\mathcal{B}_{\mathrm{PDG}}\right)$. This is not consistent with the theoretical prediction under an assumption of a pure $1^{3} D_{1}$ state [5]. We also determine the product of branching fraction $\mathcal{B}\left(D^{0} \rightarrow\left[K_{2}^{*}(1980)^{-} \rightarrow K^{-} \eta\right] \pi^{+}\right)=\left(2.2_{-1.9}^{+1.7}\right) \times 10^{-4}$. For $a_{0}(980)^{+}$, we confirm the $\pi \eta^{\prime}$ contribution in the three-channel Flatté model with a statistical significance of $10.1 \sigma$. We have also determined the branching fraction $\mathcal{B}\left(D^{0} \rightarrow \bar{K}^{*}(892)^{0} \eta\right)=\left(1.41_{-0.12}^{+0.13}\right) \%$, which is consistent with, and more precise than, the current world average of $(1.02 \pm 0.30) \%$. It deviates from the various theoretical predictions of $(0.51-0.92) \%$ [6] with a significance of more than $3 \sigma$.

\section{Measurement of Branching Fractions of $\Lambda_{c}^{+} \rightarrow \eta \Lambda \pi^{+}, \eta \Sigma^{0} \pi^{+}, \Lambda(1670) \pi^{+}$, and $\eta \Sigma(1385)^{+}$}

The branching fractions of weakly decaying charmed baryons provide a way to study both strong and weak interactions. The $\Lambda_{c}^{+} \rightarrow \eta \Lambda \pi^{+}$decay mode is especially interesting since it has been suggested that it is an ideal decay mode to study the $\Lambda(1670)$ and $a_{0}(980)$ because, for any combination of two particles in the final state, the isospin is fixed. Based on a $980 \mathrm{fb}^{-1}$ data sample, the branching fractions of $\Lambda_{c}^{+} \rightarrow \eta \Lambda \pi^{+}, \eta \Sigma^{0} \pi^{+}, \Lambda(1670) \pi^{+}$, and $\eta \Sigma(1385)^{+}$are measured [7]. The $M\left(\eta \Lambda \pi^{+}\right)$spectrum is shown in Fig. 3 (a). The $\Lambda_{c}^{+} \rightarrow \eta \Sigma^{0} \pi^{+}$is observed indirectly as a feeddown component and it has efficiency-corrected yield $N_{\text {cor }}=(3.05 \pm 0.16) \times 10^{5}$. Considering 

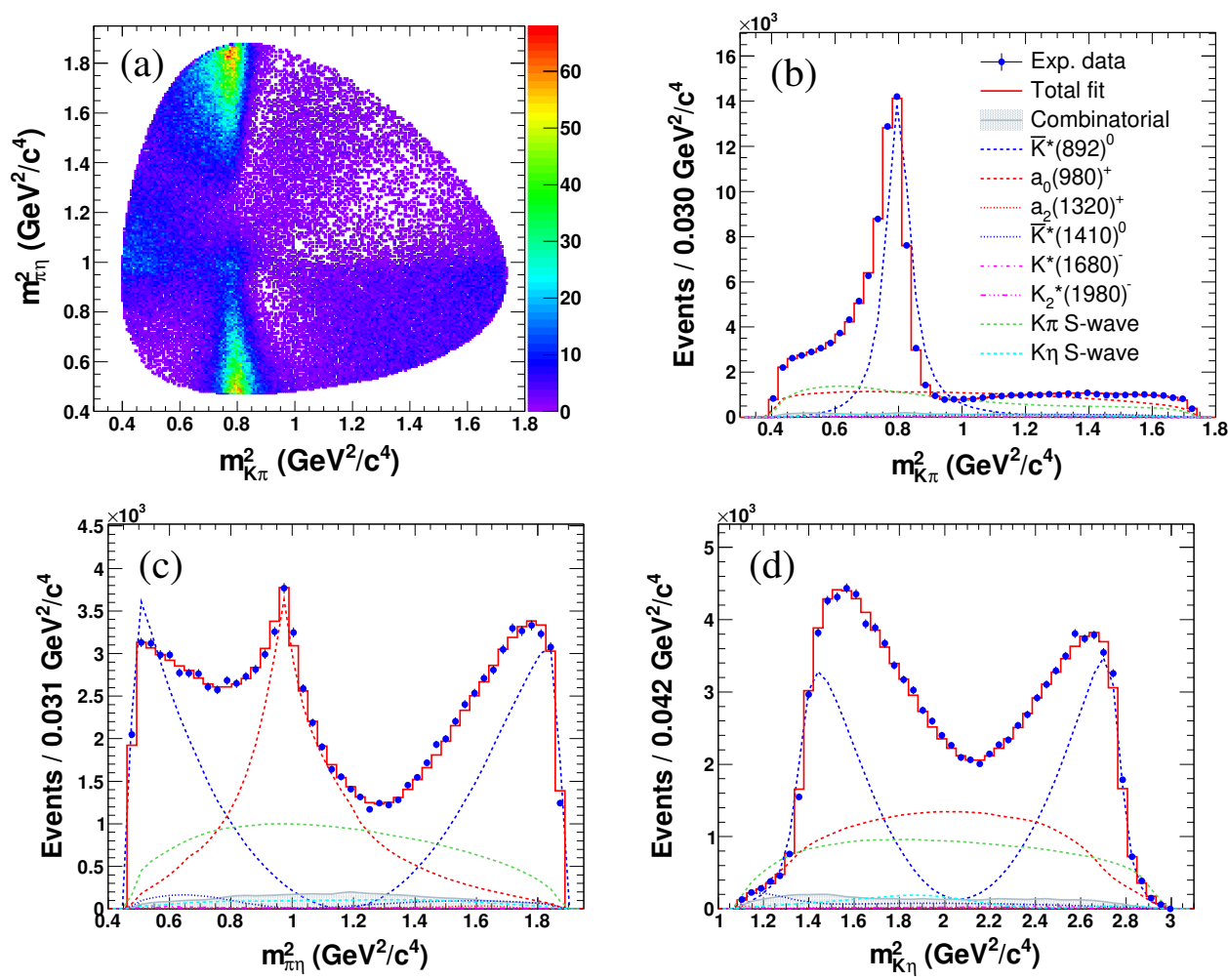

Figure 2: The Dalitz plot of $D^{0} \rightarrow K^{-} \pi^{+} \eta$ in (a) $M-Q$ signal region $1.85 \mathrm{GeV} / c^{2}<M<1.88 \mathrm{GeV} / c^{2}$ and $5.35 \mathrm{MeV} / c^{2}<Q<6.35 \mathrm{MeV} / c^{2}$, and projections on (b) $m_{K \pi}^{2}$, (c) $m_{\pi \eta}^{2}$ and (d) $m_{K \eta}^{2}$. In projections the fitted contributions of individual components are shown, along with contribution of combinatorial background (grey-filled) from sideband region.

$\Lambda_{c}^{+} \rightarrow \eta \Lambda \pi^{+}$and $\Lambda_{c}^{+} \rightarrow p K^{-} \pi^{+}$have sufficiently large statistic, the yields in individual bins of Dalitz plots are determined: $N_{c o r}\left(\eta \Lambda \pi^{+}\right)=(7.41 \pm 0.07) \times 10^{5}$ and $N_{\text {cor }}\left(p K^{-} \pi^{+}\right)=(1.005 \pm 0.001) \times 10^{7}$. Finally, the branching ratios of $\Lambda_{c}^{+} \rightarrow \eta \Lambda \pi^{+}$and $\Lambda_{c}^{+} \rightarrow \eta \Sigma^{0} \pi^{+}$relative to $\Lambda_{c}^{+} \rightarrow p K^{-} \pi^{+}$are $0.293 \pm 0.003 \pm 0.014$ and $0.120 \pm 0.006 \pm 0.006$, where the uncertainties are statistical and systematic, respectively.

On the Dalitz plot of $\Lambda_{c}^{+} \rightarrow \eta \Lambda \pi^{+}$shown in Fig. 3 (b), bands corresponding to $\Lambda_{c}^{+} \rightarrow$ $\Lambda(1670) \pi^{+} / \eta \Sigma(1385)^{+}$resonant sub-channels are seen clearly, along with $\Lambda_{c}^{+} \rightarrow \Lambda a_{0}(980)^{+}$. For every $2 \mathrm{MeV} / c^{2}$ bin of $M(\eta \Lambda)$ and $M\left(\Lambda \pi^{+}\right)$distributions, the $\Lambda_{c}^{+}$yield is obtained by fitting $M\left(\eta \Lambda \pi^{+}\right)$. Then, a relativistic Breit-Wigner with momentum-dependent width is used to describe the S-wave $\Lambda(1670)$ and the P-wave $\Sigma(1385)$, as shown in Fig. 3 (c, d). Then, we determine the relative branching ratio $\frac{\mathcal{B}\left(\Lambda_{c}^{+} \rightarrow[\Lambda(1670) \rightarrow \eta \Lambda] \pi^{+}\right)}{\mathcal{B}\left(\Lambda_{c}^{+} \rightarrow p K^{-} \pi^{+}\right)}=(5.54 \pm 0.29 \pm 0.73) \%$ and $\frac{\mathcal{B}\left(\Lambda_{c}^{+} \rightarrow \eta \Sigma(1385)^{+}\right)}{\mathcal{B}\left(\Lambda_{c}^{+} \rightarrow p K^{-} \pi^{+}\right)}=$ $0.192 \pm 0.006 \pm 0.016$. Finally after using the world averaged $\mathcal{B}\left(\Lambda_{c}^{+} \rightarrow p K^{-} \pi^{+}\right)$, we have $\mathcal{B}\left(\Lambda_{c}^{+} \rightarrow\right.$ $\left.[\Lambda(1670) \rightarrow \eta \Lambda] \pi^{+}\right)=(3.48 \pm 0.19 \pm 0.46 \pm 0.18) \times 10^{-3}$ and $\mathcal{B}\left(\Lambda_{c}^{+} \rightarrow \eta \Sigma(1385)^{+}\right)=(1.21 \pm$ $0.04 \pm 0.10 \pm 0.06) \%$, where the first two uncertainties are statistical and systematic uncertainties, and the third uncertainty is from $\mathcal{B}\left(\Lambda_{c}^{+} \rightarrow p K^{-} \pi^{+}\right)$. 

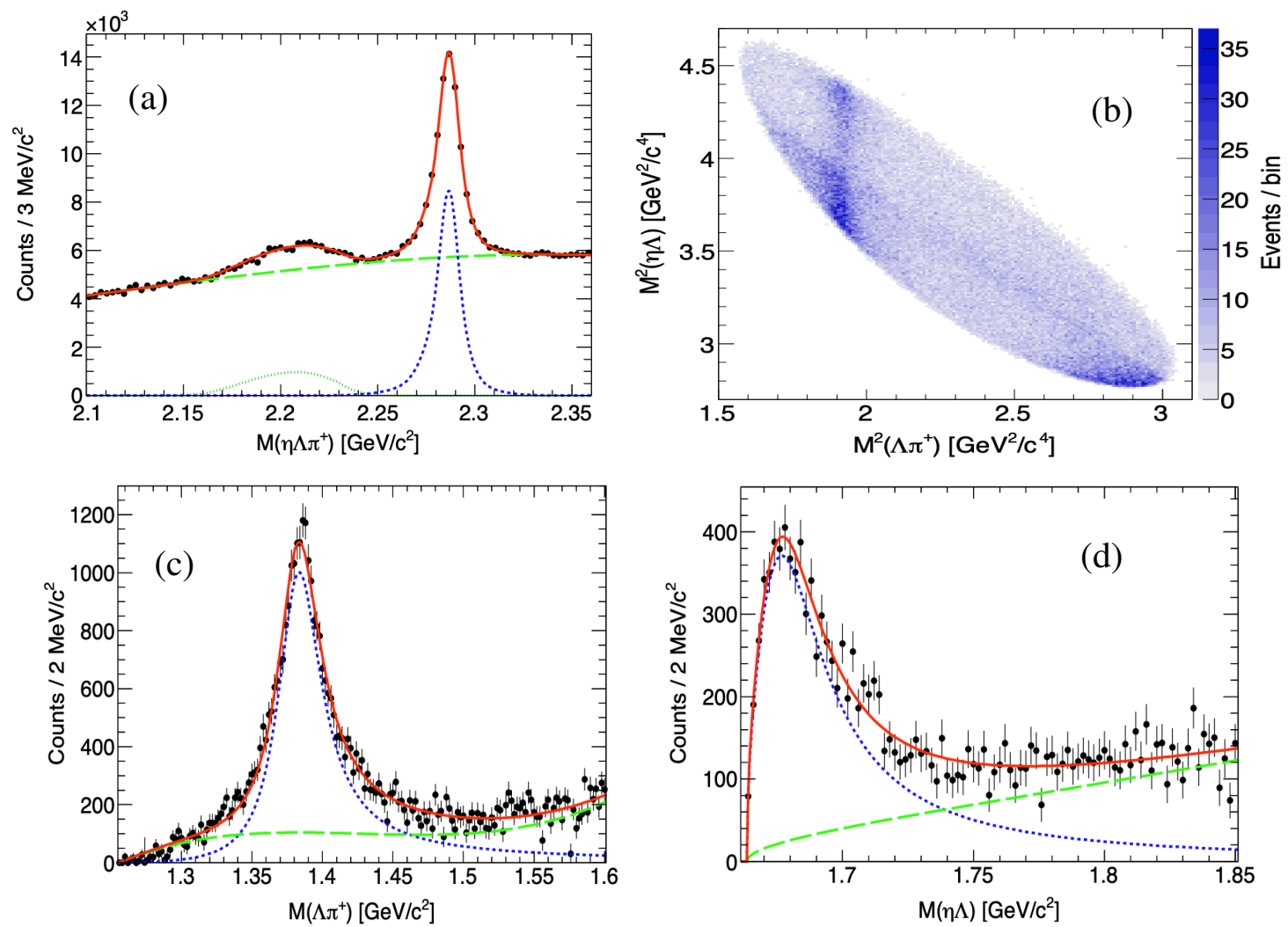

Figure 3: Top figures are (a) the invariant mass of $\eta \Lambda \pi^{+}$and (b) its Dalitz plot in signal region. Bottom figures are fits to the $\Lambda_{c}^{+}$yield in the (c) $M(\eta \Lambda)$ and (d) $M\left(\Lambda \pi^{+}\right)$spectra, where the curves indicate the total fit result (solid red), the signal modeled with a relativistic Breit-Wigner function (dashed blue), and the background (long-dashed green).

\section{First determination of the Spin and Parity of $\Xi_{c}(2970)^{+}$}

The unclear theoretical situation motivates an experimental determination of spin and parity of a charmed-strange baryon $\Xi_{c}(2970)$, which provides important information to test predictions and help decipher its nature. Using a $980 \mathrm{fb}^{-1}$ data sample, the spin and parity of a charmed-strange baryon $\Xi_{c}(2970)^{+}$is measured [8] by (1) studies of the helicity angle distributions, $\theta_{h}$ of $\Xi_{c}(2970)^{0}$ and $\theta_{c}$ of $\Xi_{c}(2645)^{0}$ in $\Xi_{c}(2970)^{+} \rightarrow \Xi_{c}(2645)^{0} \pi^{+} \rightarrow \Xi_{c}^{+} \pi^{-} \pi^{+}$, and (2) a measurement of the $\Xi_{c}(2970)^{+}$decay branching ratio $R=\mathcal{B}\left(\Xi_{c}(2970)^{+} \rightarrow \Xi_{c}(2645)^{0} \pi^{+}\right) / \mathcal{B}\left(\Xi_{c}(2970)+\rightarrow \Xi_{c}^{\prime 0} \pi^{+}\right)$.

The angular distribution are obtained by dividing the data into 10 equal bins for $\cos \theta_{h}$ and $\cos \theta_{c}$, each within intervals of 0.2 . The yield of $\Xi_{c}(2970)^{+} \rightarrow \Xi_{c}(2645)^{0} \pi^{+}$for each bin is obtained by fitting the invariant-mass distribution of $M\left(\Xi_{c}^{+} \pi^{-} \pi^{+}\right)$for the $\Xi_{c}(2645)^{0}$ signal region (within $5 \mathrm{MeV} / c^{2}$ of $\Xi_{c}(2645)^{0}$ nominal mass) and sidebands (interval from 15 to $25 \mathrm{MeV} / c^{2}$ away from $\Xi_{c}(2645)^{0}$ nominal mass). The background-subtracted and efficiency-corrected yield distribution in Fig. 4 is fitted with expected decay-angle distributions $W_{J}$ for different spin hypotheses. The best fit is for $J=1 / 2$, while others are excluded with small significance, which shows inconclusive result. For helicity angle $\theta_{c}$, with an assumption that the lowest partial wave dominates, the expected angular correlation $W\left(\theta_{c}\right)$ is used to describe the distribution. Finally the $J^{P}=1 / 2^{+}$hypothesis is 
better than $3 / 2^{-}$or $5 / 2^{+}$ones at the level of $5.1 \sigma$ or $4.0 \sigma$.
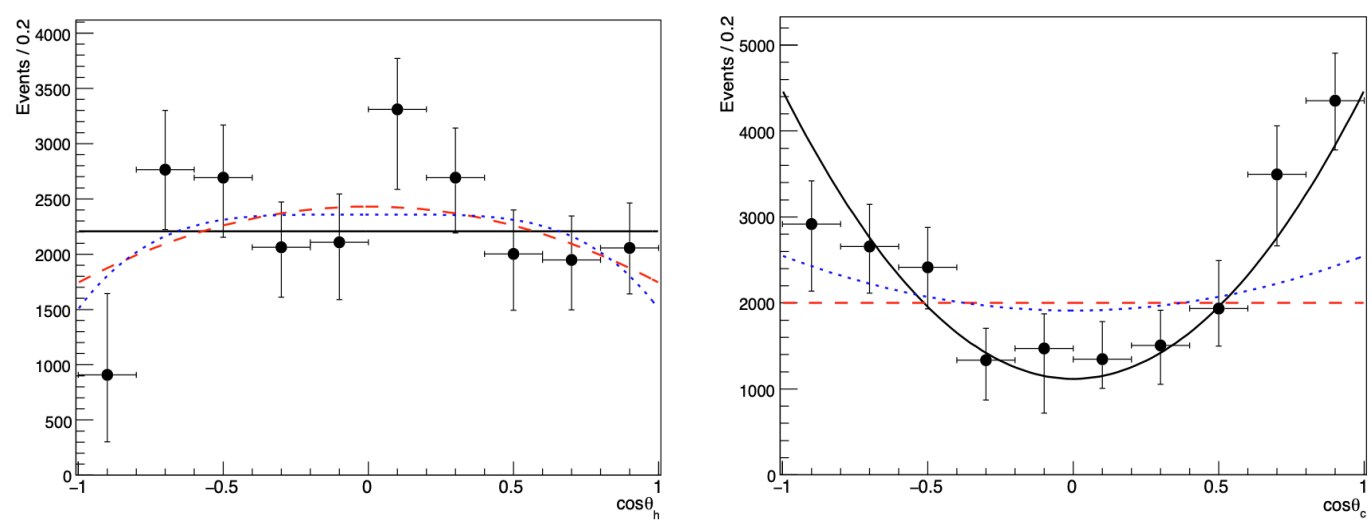

Figure 4: The yields on the cosine of helicity angle of $\Xi_{c}(2970)^{0}$ (left, $J=1 / 2$ for solid black; $J=3 / 2$ for dashed red; $J=5 / 2$ for dotted blue) and on cosine of helicity angle of $\Xi_{c}(2645)^{0}$ (right, $J^{P}=1 / 2^{+}$for solid black; $J=3 / 2^{-}$for dashed red; $J=5 / 2^{+}$for dotted blue) in $\Xi_{c}(2970)^{+} \rightarrow \Xi_{c}(2645)^{0} \pi^{+}$decay.

The parity of $\Xi_{c}(2970)^{+}$is established [8] from the ratio between $\mathcal{B}\left(\Xi_{c}(2970)^{+} \rightarrow \Xi_{c}(2645)^{0} \pi^{+}\right)$ and $\mathcal{B}\left(\Xi_{c}(2970)^{+} \rightarrow \Xi_{c}^{\prime 0} \pi^{+}\right)$by $R=\frac{N^{*}}{\epsilon^{*} N\left(\Xi_{c}^{+}\right) / \epsilon^{+}} / \frac{N^{\prime}}{\sum_{i} \epsilon_{i}^{\prime} N\left(\Xi_{c}^{0}\right)_{i} / \epsilon_{i}^{0}}$, where $\Xi_{c}^{0}$ uses two modes, $\Xi^{-} \pi^{+}$ and $\Omega^{-} K^{+}$. The yields $N^{*, \prime}$ are obtained by fitting the invariant-mass distributions in Fig. 5. Finally we have $R=1.67 \pm 0.29$ (stat $)_{-0.09}^{+0.15}$ (syst) \pm 0.25 (IS), where the last uncertainty is due to possible isospin-symmetry-breaking effects (15\%). Heavy-quark spin symmetry (HQSS) predicts $R=1.06$ (0.26) for a $1 / 2^{+}$state with the spin of the light-quark degrees of freedom $s_{l}=0$ (1) [9]. Our result favors a positive-parity assignment with $s_{l}=0$. We note that HQSS predictions could be larger than the quoted value by a factor of $\sim 2$ with higher-order terms in $\left(1 / m_{c}\right)$ [10], so our result is consistent with the HQSS prediction for $J^{P}\left(s_{l}\right)=1 / 2^{+}(0)$.
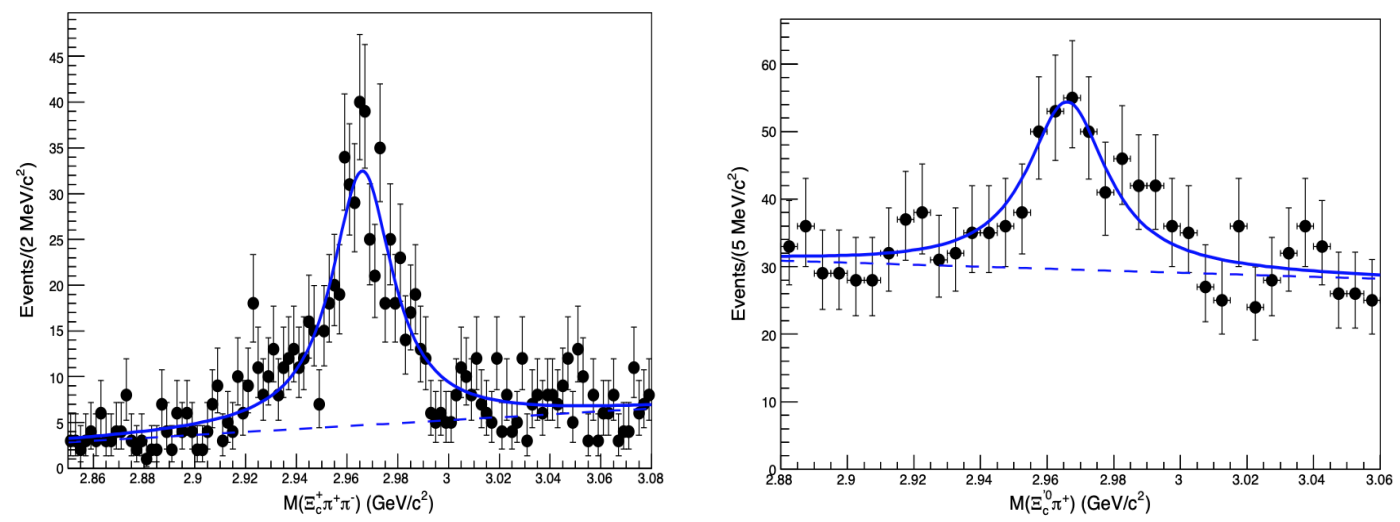

Figure 5: $\Xi_{c}^{+} \pi^{-} \pi^{+}$invariant-mass distribution for $\Xi_{c}(2970)^{+} \rightarrow \Xi_{c}(2645)^{0} \pi^{+} \rightarrow \Xi_{c}^{+} \pi^{-} \pi^{+}$, and $\Xi_{c}^{\prime 0} \pi^{+}$ invariant-mass distribution for $\Xi_{c}(2970)^{+} \rightarrow \Xi_{c}^{\prime 0} \pi^{+} \rightarrow \Xi_{c}^{0} \gamma \pi^{+}$. The fit result (solid blue curve) is presented along with the background (dashed blue curve)

\section{Summary}

Belle experiment has achieved the fruitful productions of flavor physics to date. Some selected recent charm results are presented, including charm mixing parameter $y_{C P}$ in $C P$-odd decay 
$D^{0} \rightarrow K_{S}^{0} \omega$, hadronic decays $D^{0} \rightarrow K^{-} \pi^{+} \eta$ and $\Lambda_{c}^{+} \rightarrow \eta \Lambda \pi^{+} / \eta \Sigma^{0} \pi^{+}$, first determination of the spin and parity of $\Xi_{c}(2970)^{+}$. More charming charm results from Belle will come out in near future. As a summary, I would like to say, "Belle is not only keeping alive but still keeping energetic, together with its upgraded experiment Belle II who is under a rapid growth."

\section{References}

[1] S. Kurokawa and E. Kikutani, Nucl. Instrum. Methods Phys. Res. Sect. A 499, 1 (2003), and other papers included in this Volume.

[2] A. Abashian et al. (Belle Collaboration), Nucl. Instrum. Methods Phys. Res. Sect. A 479, 117 (2002).

[3] M. Nayak et al. (Belle Collaboration), Phys. Rev. D 102, 071102(R) (2020).

[4] Y. Q. Chen et al. (Belle Collaboration), Phys. Rev. D 102, 012002 (2020).

[5] T. Barnes, N. Black, and P. R. Page, Phys. Rev. D 68, 054014 (2003); C. Q. Pang, J. Z. Wang, X. Liu, et al. Eur. Phys. J. C (2017) 77: 861.

[6] H. Y. Cheng and C. W. Chiang, Phys. Rev. D 81, 074021 (2010); H. N. Li, C. D. Lü, and F. S. Yu, Phys. Rev. D 86, 036012 (2012); Q. Qin, H. N. Li, C. D. Lü, and F. S. Yu, Phys. Rev. D 89, 054006 (2014).

[7] J. Y. Lee et al. (Belle Collaboration), arXiv:2008.11575.

[8] T. J. Moon et al. (Belle Collaboration), arXiv:2007.14700

[9] Hai-Yang Cheng and Chun-Khiang Chua, Phys. Rev. D 75, 014006 (2007).

[10] Adam F. Falk and Thomas Mehen, Phys. Rev. D 53, 231 (1996). 